\title{
Glándula pediosa de moluscos terrestres y sus implicancias evolutivas, con énfasis en Megalobulimus
}

\section{Pediose gland in land snails and its evolutionary implications, with emphasis on Megalobulimus}

\author{
Victor Borda ${ }^{1,2}$, Rina Ramírez ${ }^{1,2}$ y Pedro Romero ${ }^{1,2}$
}

1 Instituto de Investigación de Ciencias Biológicas Antonio Raimond (ICBAR). Facultad de Ciencias Biológicas, Universidad Nacional 2 Museo de Historia Natural, Universidad Nacional Mayor de San Marcos. Av. Arenales 1256, Apartado 14-0434, Lima-14, Perú.

Email Victor Borda: vic_bp@yahoo.es Email Rina Ramírez: rina_rm@yahoo.com

Trabajo presentado a la XVIII Reunión Científica del Instituto de Investigaciones en Ciencias Biológicas Antonio Raimondi, "200 años del nacimiento de Charles Darwin y el 150 aniversario de la publicación de On the Origin of Species by Means of Natural Selection". Del 19 al 21 de agosto de 2009 .

Publicado impreso: 20/10/2010 Publicado online: 29/09/2010

\section{Resumen}

Se describe la anatomía de la glándula pediosa de cinco especies de Megalobulimus (Megalobulimidae), y son comparadas con gastrópodos pulmonados Succineidae, Orthalicidae, Helicidae y Veronicellidae. Una sinapomorfía considerada para el clado Stylommatophora es la presencia de una membrana que aísla a la glándula pediosa de la cavidad visceral. Las especies estudiadas mostraron un grado variable de sujeción de la glándula al pie, desde apenas sujeta por escasa fibras (Megalobulimus) hasta aquella totalmente aislada por una membrana (Cantareus) pasando por grados intermedios (Succinea y Bostryx). La glándula pediosa en la seudobabosa Heterovaginia limayana (Systellommatophora) no está adherida al piso de la cavidad visceral. En Megalobulimus, la parte glandular es voluminosa y pende del techo de la cápsula, a diferencia de las otras especies en que los acinos glandulares están uniformemente adosados a la pared interna de la cápsula. Se describen nuevos caracteres para la glándula pediosa que mejoran la diagnosis del género Megalobulimus y su evaluación filogenética.

Palabras claves: Megalobulimidae, Succinea peruviana, Bostryx conspersus, Heterovaginina limayana, sinapomorfía.

\section{Abstract}

We describe the anatomy of pediose gland in five species of Megalobulimus (Megalobulimidae) and contrast them with those of succineid, orthalicid and helicid gastropods. The presence of a membrane that isolates the pediose gland from the visceral cavity is a synapomorhy of the Stylommatophora clade. A variable range of fixation of the gland to the muscular foot is observed in studied species, from a gland barely held by few fibers (Megalobulimus) to a totally isolated gland (Cantareus), passing through different intermediate grades (Succinea and Bostryx). The pediose gland in Heterovaginina limayana (Systelomatophora) is not attached to the bottom of the visceral cavity. The glandular portion is a voluminous structure that dangles from the capsule roof in Megalobulimus, whereas in other species it is attached to the capsule internal wall. We describe new pediose gland characteristics that reinforce diagnosis of the genus Megalobulimus and provide more phylogenetic information.

Keywords: Megalobulimidae, Succinea peruviana, Bostryx conspersus, Heterovaginina limayana, synapomorphy.

\section{Introducción}

Las dos sinapomorfías que sustentan al clado Stylommatophora son los tentáculos retráctiles y la presencia de una membrana que aísla la glándula pediosa de la cavidad visceral. En contraste, los Systellommatophora presentan tentáculos contráctiles y la glándula pediosa libre en la cavidad visceral (Dayrat \& Tillier 2002 y 2003).

La glándula pediosa, también llamada glándula pedal o suprapediosa (Barker 2001), es un órgano propio de los gastrópodos, de origen ectodérmico (Chandra 1962). Corresponde a una glándula del tipo exocrino; está encargada de producir la baba, sustancia mucosa que le ayuda en la locomoción y además provee al animal de protección frente a la pérdida de humedad (Barr 1926). La baba de caracol está compuesta por alantoína, vitaminas, colágeno, elastina, ácido glicólico (Bascuñan 1992, apud Cáceres 2006), mucopolisacáridos y agua (Levene 1925). En los últimos tiempos, la baba de caracol ha tomado gran importancia comercial, por supuestas propiedades cosméticas, con comprobado efecto humectante (Cáceres 2006).

La morfología e histología de esta glándula es poco conocida. La referencia más detallada proviene de André (1894), es para especies europeas. En América del sur, la única descripción detallada corresponde a Hylton-Scott (1939) realizada en Megalobulimus lorentzianus; otras descripciones se refieren a la apariencia externa (Thomé et al. 1994, Pena et al. 2004).
El objetivo del presente trabajo es evaluar las características morfológicas de la glándula pediosa de cinco especies de Megalobulimus y compararlas con otras de cuatro especies de diferente posición evolutiva (Succinea, Bostryx, Cantareus y Heterovaginina), en la búsqueda de caracteres que mejoren la diagnosis y el análisis filogenético del género Megalobulimus.

\section{Materiales y métodos}

Se utilizaron especímenes adultos de cinco especies de $\mathrm{Me}$ galobulimus de distintos departamentos de Perú. La especie $M$. capillaceus (Pfeiffer, 1885) (Fig. 1A) es la que presenta la concha de menor tamańo (altura: $58-77 \mathrm{~mm}$ ); tiene el labio rojo por lo que es conocido con el nombre vulgar de "pucashimi" en el Departamento de San Martín (Ramírez \& Cáceres 1991). La siguiente en tamaño es $M$. lichtensteini (Albers, 1854) (Fig. 1B) (altura: 72-86 mm), procedente del Dpto. Amazonas. Las restantes tres especies tienen las conchillas más grandes y son conocidas con el nombre vulgar de "congompe": M. maximus (Sowerby, 1825) (Fig. 1C) (altura: 106-154 mm) у $M$. thammianus (Martens, 1876) (Fig. 1D) (altura: 88-116 mm) procedentes del Dpto. Madre de Dios y M. popelairianus (Nyst, 1845) (Fig. 1E) (altura: 110-163 mm) de los Dptos. Madre de Dios y San Martín.

También se emplearon especímenes adultos de Succinea peruviana (Philippi, 1867) (Fig. 1F) y Bostryx conspersus (Sowerby, 1833) (Fig. 1G) procedentes del ecosistema de Lomas del Dpto. 

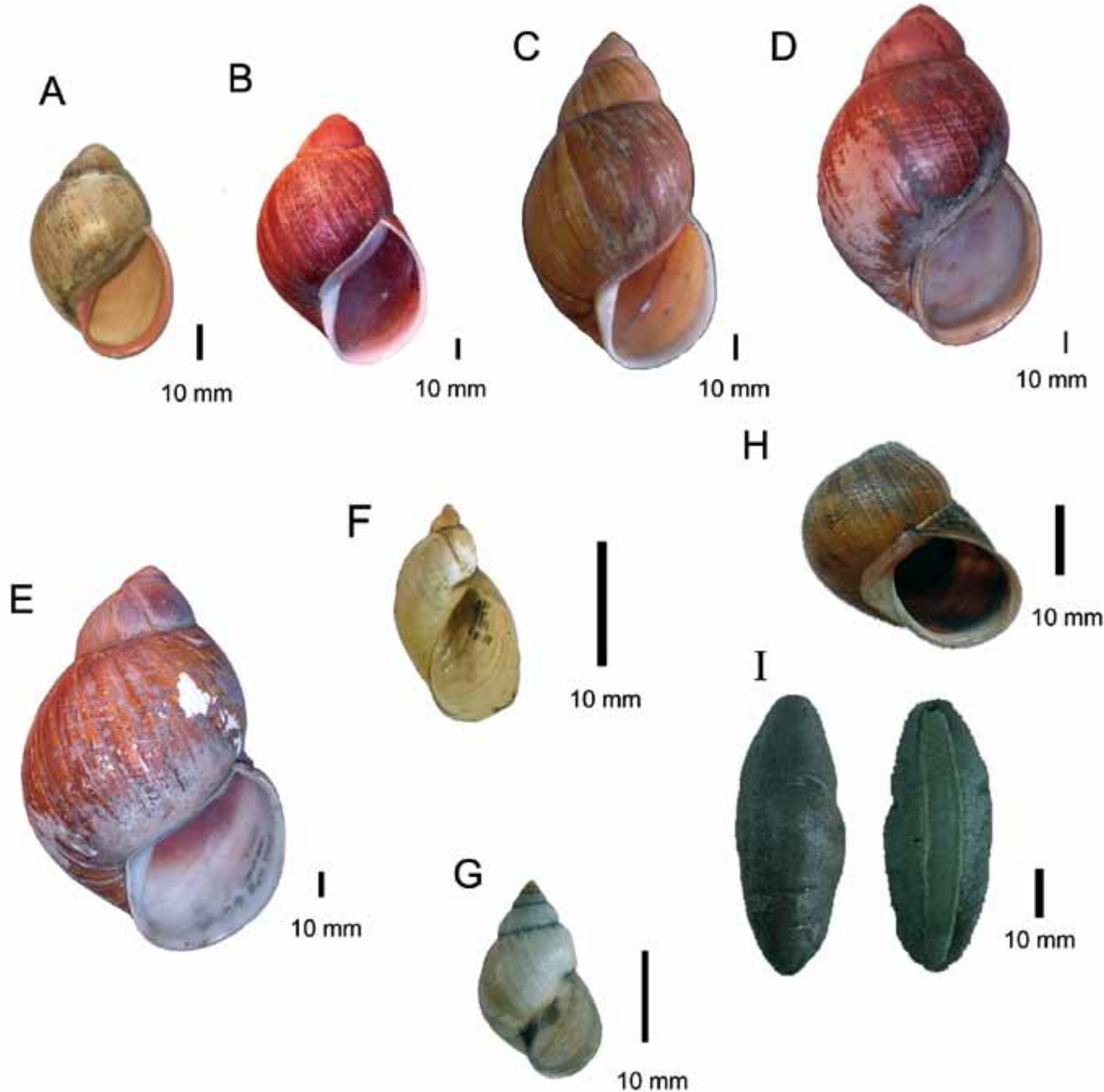

$10 \mathrm{~mm}$

Figura 1. Conchas e individuos de las especies estudiadas. (A) Megalobulimus capillaceus. (B) M. lichtensteini. (C) M. maximus. (D) M. thammianus. (E) M. popelairianus. (F) Succinea peruviana. (G) Bostryx conspersus. (H) Cantareus aspersus. (I) Heterovaginina limayana.

Lima y de Cantareus aspersus (Müller, 1774) (=Helix aspersa) (Fig. 1H) y la seudobabosa Heterovaginina limayana (Lesson, 1829) (Fig. 1I) procedentes del jardín del Museo de Historia Natural de la Universidad Nacional Mayor de San Marcos (MUSM) (Tabla 1). El material estudiado se encuentra custodiado en la Colección Malacológica del Departamento de Malacología y Carcinología del MUSM.

Los ejemplares fueron sacrificados por ahogamiento en recipientes con agua herméticamente cerrados por 24 horas. Los ejemplares fueron fijados y preservados en etanol 96. Algunos especímenes fijados fueron inyectados con azul de metileno por la abertura externa de la glándula pediosa, para una mejor visualización de las estructuras. Las disecciones se iniciaron, en el caso de caracoles, mediante un corte longitudinal en el lado derecho de la pared del cuerpo. En la cavidad visceral, se levantó el bulbo bucal y se separó cuidadosamente las ramificaciones de los ganglios cerebroides (Beaumont \& Cassie 1970), visualizándose así la glándula pediosa. En las seudobabosas, las disecciones fueron realizadas a partir de un corte por el surco pedal del lado derecho (Thomé \& Lopes 1973). La parte interna de la glándula se dejó al descubierto mediante un corte longitudinal en su pared lateral derecha. Las estructuras fueron analizadas al estereomicoscopio y fotografiadas con una cámara digital. Se tomaron las medidas del largo y ancho máximo de la glándula pediosa, además de algunas características internas de la glándula, así como del largo y ancho del pie de los individuos analizados, empleando un calibrador vernier.

\section{Resultados}

En las especies estudiadas, la glándula pediosa se abre al exterior mediante una abertura ubicada en el fondo de un repliegue 
Tabla 1. Datos de colecta y material biológico analizado en el presente estudio

\begin{tabular}{|c|c|c|c|c|c|}
\hline Especie & No. ind. & Procedencia & Coordenadas & $\begin{array}{l}\text { Altitud } \\
(\mathrm{m})\end{array}$ & Colector \\
\hline \multicolumn{6}{|c|}{ Phylum Mollusca, Clase Gastropoda, Subclase Pulmonata, Orden Eupulmonata, Suborden Stylommatophora } \\
\hline \multicolumn{6}{|c|}{ Infraorden Sigmurethra, Familia Megalobulimidae } \\
\hline Megalobulimus capillaceus & 5 & Dpto. San Martín: Juan Guerra & $\begin{array}{l}06^{\circ} 35^{\prime} 16^{\prime \prime} \mathrm{S} \\
76^{\circ} 18^{\prime} 50^{\prime \prime} \mathrm{W}\end{array}$ & 237 & $\begin{array}{l}\text { R. Ramírez, C. Calderón \& V. } \\
\text { Borda. } 2008\end{array}$ \\
\hline Megalobulimus lichtensteini & 2 & Dpto. Amazonas: Naranjillo & $\begin{array}{l}05^{\circ} 49^{\prime} 19,2^{\prime \prime} \mathrm{S} \\
78^{\circ} 16^{\prime} 30,6^{\prime \prime} \mathrm{W}\end{array}$ & 489 & V. Borda \& C. Calderón. 2008 \\
\hline Megalobulimus thammianus & 2 & $\begin{array}{c}\text { Dpto. Madre de Dios: Reserva Amazónica } \\
\text { INKATERRA }\end{array}$ & $12^{\circ} 35^{\prime} \mathrm{S}, 69^{\circ} 05^{\prime} \mathrm{W}$ & 200 & $\begin{array}{l}\text { R. Ramírez, V. Borda \& P. } \\
\text { Romero. } 2008-2009\end{array}$ \\
\hline \multirow[t]{2}{*}{ Megalobulimus maximus } & 2 & $\begin{array}{c}\text { Dpto. Madre de Dios: Reserva Amazónica } \\
\text { INKATERRA }\end{array}$ & $12^{\circ} 35^{\prime} \mathrm{S}, 69^{\circ} 05^{\prime} \mathrm{W}$ & 200 & V. Borda \& P. Romero. 2008 \\
\hline & & $\begin{array}{l}\text { Dpto. Madre de Dios: Comunidad Nativa } \\
\text { "El infierno" }\end{array}$ & $\begin{array}{l}12^{\circ} 46^{\prime} 15,10^{\prime \prime} \mathrm{S} \\
69^{\circ} 14^{\prime} 28,70^{\prime \prime} \mathrm{W}\end{array}$ & 198 & C. Díaz. 1988 \\
\hline \multirow[t]{2}{*}{ Megalobulimus popelairianus } & 3 & $\begin{array}{l}\text { Dpto. Madre de Dios: Reserva Amazónica } \\
\text { INKATERRA (ex Cuzco Amazónico) ( } 2 \text { ind) }\end{array}$ & $12^{\circ} 35^{\prime} \mathrm{S}, 69^{\circ} 05^{\prime} \mathrm{W}$ & 200 & R. Ramírez. 1988 \\
\hline & & Dpto. San Martín: Saposoa-Chambira (1 ind) & $\begin{array}{l}06^{\circ} 56^{\prime} 07,2^{\prime \prime} \mathrm{S} \\
76^{\circ} 46^{\prime} 16,2^{\prime \prime} \mathrm{W}\end{array}$ & 311 & A. Ruiz \& R. Ramírez. 2008 \\
\hline \multicolumn{6}{|c|}{ Infraorden Sigmurethra, Familia Orthalicidae } \\
\hline Bostryx conspersus & 4 & Dpto. Lima: Lomas “El Paraiso” & $\begin{array}{l}12^{\circ} 08^{\prime} 20^{\prime \prime} \mathrm{S} \\
76^{\circ} 55^{\prime} 23^{\prime \prime} \mathrm{W}\end{array}$ & 500 & R. Ramírez. 2001 \\
\hline \multicolumn{6}{|c|}{ Infraorden Sigmurethra, Familia Helicidae } \\
\hline Cantareus aspersus & 5 & $\begin{array}{c}\text { Dpto. Lima: Jardín del Museo de Historia } \\
\text { Natural }\end{array}$ & $\begin{array}{l}12^{\circ} 04^{\prime} 38,3^{\prime \prime} \mathrm{S} \\
77^{\circ} 02^{\prime} 12.6^{\prime \prime} \mathrm{W}\end{array}$ & 122 & V. Borda. 2009 \\
\hline \multicolumn{6}{|c|}{ Infraorden Elasmognatha, Familia Succineidae } \\
\hline Succinea peruviana & 4 & Dpto. Lima: Lomas “El Lúcumo” & $\begin{array}{l}12^{\circ} 15^{\prime} 22^{\prime \prime} \mathrm{S} \\
76^{\circ} 47^{\prime} 16^{\prime \prime} \mathrm{W}\end{array}$ & 400 & R. Ramírez. 2001 \\
\hline \multicolumn{6}{|c|}{ Phylum Mollusca, Clase Gastropoda, Subclase Pulmonata, Orden Eupulmonata, } \\
\hline \multicolumn{6}{|c|}{ Suborden SystellommatophoraFamilia Veronicellidae } \\
\hline Heterovaginina limayana & 5 & $\begin{array}{c}\text { Dpto. Lima: Jardín del Museo de Historia } \\
\text { Natural }\end{array}$ & $\begin{array}{l}12^{\circ} 04^{\prime} 38,3^{\prime \prime} \mathrm{S} \\
77^{\circ} 02^{\prime} 12,6^{\prime \prime} \mathrm{W}\end{array}$ & 122 & V. Borda. 2009 \\
\hline
\end{tabular}

Tabla 2. Medidas básicas de la glándula pediosa en las diferentes especies de moluscos terrestres estudiadas.

\begin{tabular}{|c|c|c|c|c|c|c|}
\hline Especie & $\begin{array}{l}\text { Largo del } \\
\text { pie (mm) }\end{array}$ & $\begin{array}{l}\text { Largo de la } \\
\text { glándula } \\
\text { (mm) }\end{array}$ & $\begin{array}{l}\text { Ancho de } \\
\text { la glándula } \\
\text { (mm) }\end{array}$ & $\begin{array}{l}\text { Surco del techo } \\
\text { de la glándula } \\
\text { (mm) }\end{array}$ & $\begin{array}{l}\text { Cresta del piso } \\
\text { de la glándula } \\
\text { (mm) }\end{array}$ & $\begin{array}{l}\text { Surco del } \\
\text { piso de la } \\
\text { glándula } \\
(\mathrm{mm})\end{array}$ \\
\hline Megalobulimus capillaceus $(\mathrm{n}=5)$ & $38,77 \pm 6,82$ & $23,45 \pm 2,37$ & $4,38 \pm 0,887$ & $14,41 \pm 3,72$ & $4,71 \pm 0,217$ & - \\
\hline M. lichtensteini $(\mathrm{n}=2)$ & $56,79 \pm 6,92$ & $24,6 \pm 0,537$ & $6,68 \pm 1,159$ & $14,86 \pm 3,082$ & $6,83 \pm 2,05$ & - \\
\hline M. thammianus $(\mathrm{n}=2)$ & $64,42 \pm 4,58$ & $18,88 \pm 1,301$ & $6,35 \pm 0,947$ & $6,36 \pm 0,961$ & - & - \\
\hline M. maximus $(\mathrm{n}=2)$ & $52,27 \pm 2,76$ & $22,66 \pm 3,62$ & $7,02 \pm 1,103$ & $4,56 \pm 0,509$ & $7,02 \pm 0,141$ & - \\
\hline M. popelairianus $(\mathrm{n}=3)$ & $83,17 \pm 11,95$ & $24,19 \pm 2,866$ & $6,54 \pm 1,981$ & $4,94 \pm 1,276$ & $8,09 \pm 3,353$ & - \\
\hline Succinea peruviana $(\mathrm{n}=4)$ & $8,09 \pm 1,02$ & $3,66 \pm 0,603$ & $1,22 \pm 0,517$ & - & - & $\approx 1,83$ \\
\hline Bostryx conspersus $(\mathrm{n}=4)$ & $14,76 \pm 1,92$ & $7,71 \pm 0,721$ & $2,2 \pm 0,65$ & - & - & $\approx 3,855$ \\
\hline Cantareus aspersus $(\mathrm{n}=5)$ & $32,29 \pm 2,87$ & $18,56 \pm 2,211$ & $2,54 \pm 0,245$ & - & - & 12,46 \\
\hline Heterovaginina limayana $(\mathrm{n}=5)$ & $44,7 \pm 6,57$ & $8,17 \pm 0,932$ & $1,97 \pm 0,15$ & - & - & - \\
\hline
\end{tabular}


entre la boca y el extremo anterior del pie. Se ubica en el eje antero-posterior del animal, debajo del bulbo bucal, entre dos ramas de nervios que provienen de los ganglios cerebroides. Entre el bulbo bucal y la glándula pediosa, en la posición más anterior, se encuentran dos haces musculares oblicuos que van de lado a lado de la pared del cuerpo y que cubren parte del tercio anterior de la glándula. Presenta las tres partes típicas de una glándula exocrina: cápsula, lumen y cuerpo glandular propiamente dicho.

\section{Características de la glándula pediosa por especies}

\section{Megalobulimus capillaceus (Figs. 2A y B)}

La glándula pediosa en $M$. capillaceus se caracteriza por ser una estructura alargada, deprimida y su longitud $(23,45 \mathrm{~mm}$ ) (Tabla 2) abarca todo el largo de la cavidad visceral. Presenta forma triangular alargada y bordes ondulados. El tercio anterior es de ancho constante que va disminuyendo hacia el extremo posterior, que es delgado y romo. Tan solo la parte dorsal de su tercio anterior, entre los dos haces de músculos oblicuos y el primer par de ramas nerviosas en relación a los ganglios cerebroides, se encuentra cubierto por un delgado tejido conectivo, quedando el resto libre hasta su extremo posterior. Su tercio posterior está sujeto al pie por escasas fibras que provienen de sus bordes laterales. Los dos haces musculares oblícuos son de menor espesor en comparación a las de otras especies de Megalobulimus aquí estudiadas.
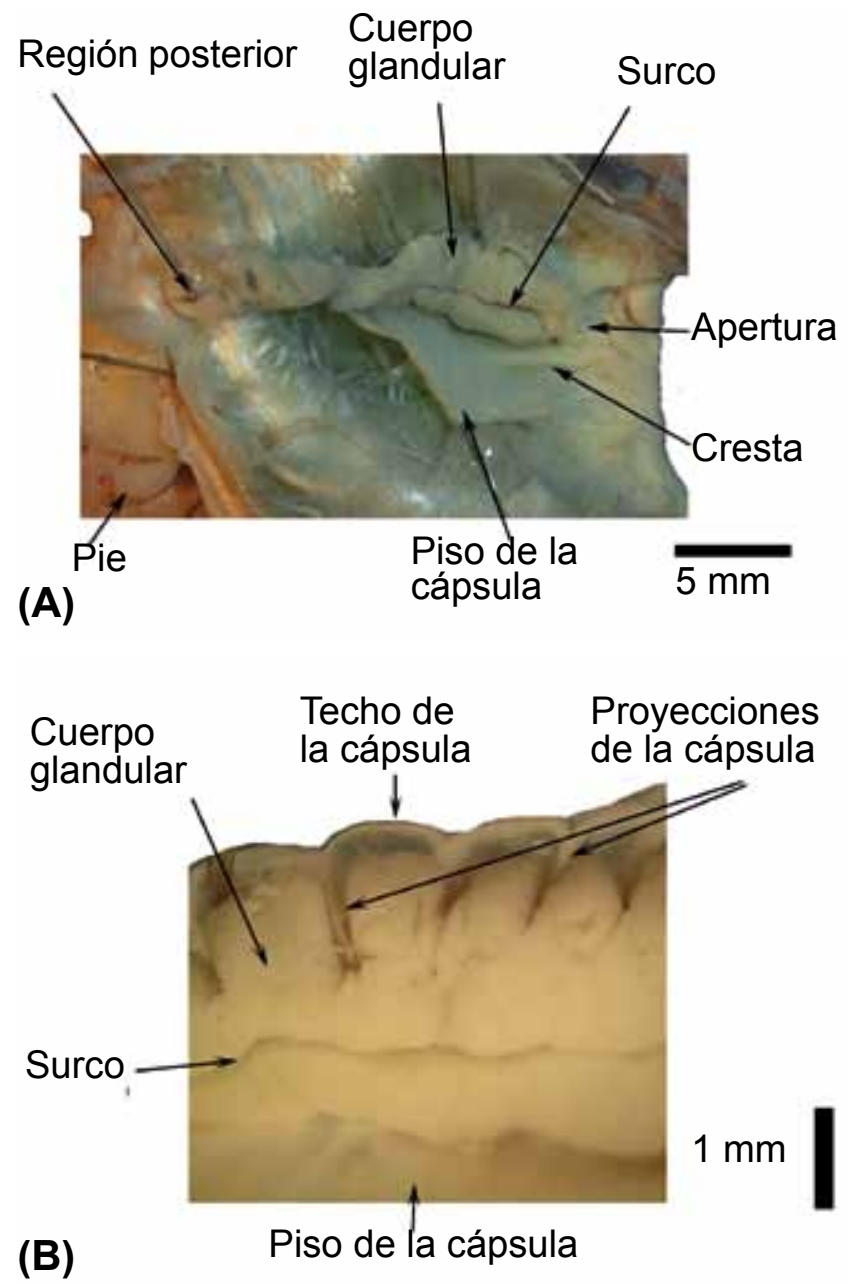

Figura 2. Glándula pediosa de Megalobulimus capillaceus. (A) Glándula parcialmente abierta, (B) Detalle del cuerpo glandular.
La cápsula de la glándula es semitransparente, por lo que el cuerpo glandular puede ser apreciado desde el exterior. El lumen es reducido, abarca toda la longitud de la glándula y su ancho varia según el ancho de ésta.

El cuerpo glandular (Fig. 2A) propiamente dicho es tan prominente que oblitera casi todo el lumen; pende del techo de la cápsula por 14-16 proyecciones sujetas al techo y sus superficies láterales (Fig. 2B). Es de color cremoso y superficie lisa, presenta un surco longitudinal medio que se extiende a todo lo largo. La profundidad del surco disminuye hacia la parte posterior. En cuatro de los cinco individuos estudiados, se observó que el surco se extendía en todo el largo de la glándula, mientras que en el otro individuo estaba interrumpido a la mitad quedando como dos surcos, uno a continuación de otro.

En el interior del cuerpo glandular se observan acinos glandulares separados por las proyecciones del techo de la cápsula.

El piso de la cápsula es liso y presenta un pequeño levantamiento a lo largo de la línea media de su tercio anterior (Fig. 2A), coincidiendo con el surco del cuerpo glandular.

\section{Megalobulimus lichtensteini (Figs. 3)}

La glándula pediosa en $M$. lichtensteini es alargada, deprimida y con bordes ondulados, su largo (24,6 mm) (Tabla 2) abarca casi toda la longitud de la cavidad visceral. Su ancho mayor se ubica entre los tercios anterior y medio de la glándula y su extremo posterior es romo y delgado. Una delgada membrana transparente cubre dorsalmente su tercio anterior, entre los dos haces de músculo oblicuo y el primer par de ramas nerviosas en relación a los ganglios cerebroides. Su tercio posterior está sujeto al pie por escasas fibras que provienen de sus bordes laterales.

La cápsula es semitransparente, que permite ver el cuerpo glandular propiamente dicho desde el exterior. El lumen tiene un ancho reducido, pero abarca toda la longitud de la glándula.

El cuerpo glandular propiamente dicho es prominente y está sujeto al techo y paredes laterales de la cápsula por delgadas proyecciones, cuyos puntos de inserción se intercalan en el borde libre colindante con la cápsula, formando entre 11 y 17 aberturas estrechas (Figs. 3). Es de color cremoso y superficie lisa, presenta un surco longitudinal que se extiende en los dos tercios anteriores de la glándula y su profundidad disminuye conforme avanza al extremo posterior. En el interior se observan acinos glandulares separados por las proyecciones del techo de la cápsula. Estas separaciones son incompletas ya que los acinos pueden llegar a tener contacto.

La porción ventral de la cápsula es delgada, pero más gruesa con respecto a la de $M$. capillaceus. El piso de la cápsula es liso y presenta a lo largo de la línea media un pequeño levantamiento que va desde la abertura hasta el tercio medio de la glándula. Ésta coincide con el surco del cuerpo glandular.

\section{Megalobulimus maximus (Figs. 4A y $\mathrm{B}$ )}

La glándula pediosa de $M$. maximus es alargada, tiene forma de daga, con el extremo posterior romo, es deprimida y tiene bordes irregulares. Su longitud $(22,66 \mathrm{~mm}$ ) (Tabla 2) abarca casi toda la longitud de la cavidad visceral, el ancho mayor se ubica 


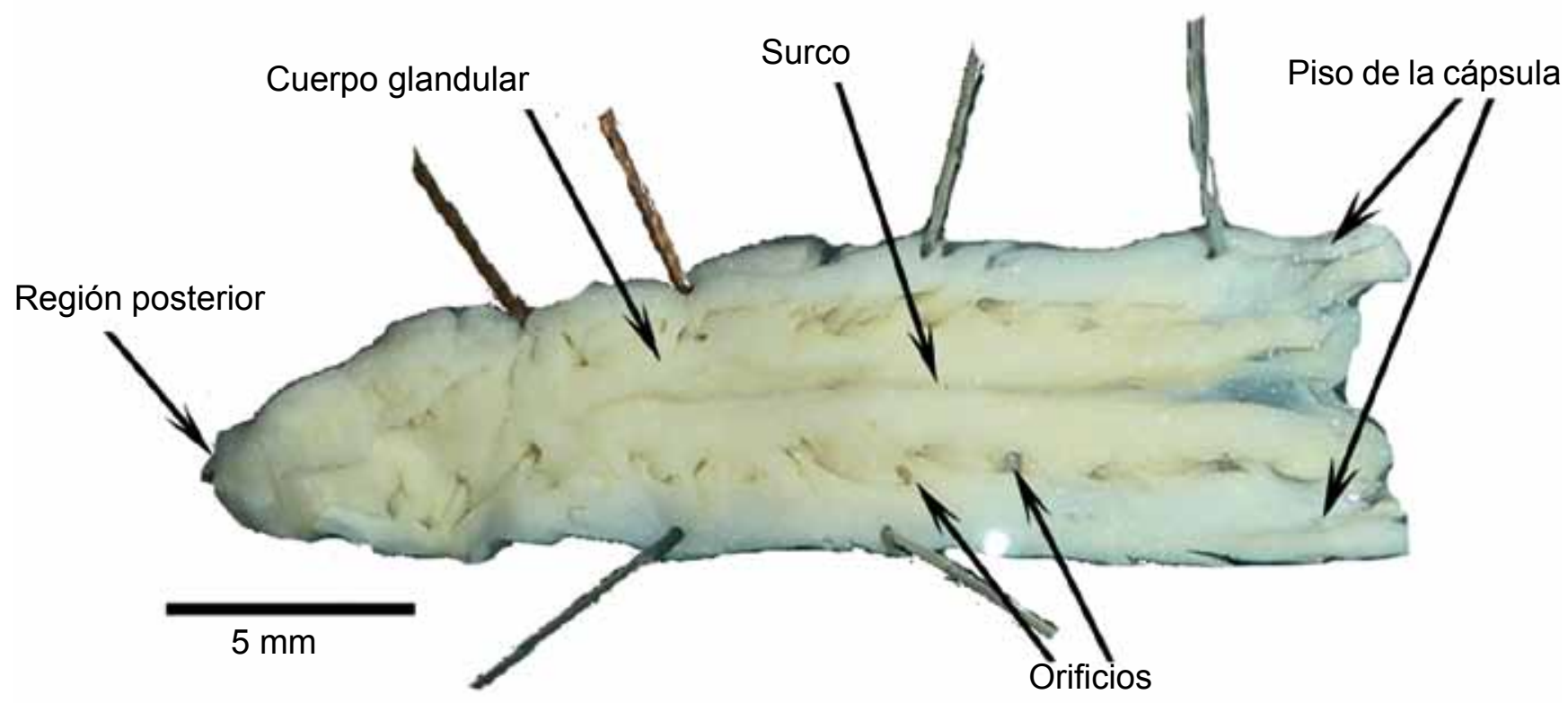

Figura 3. Glándula pediosa de Megalobulimus lichtensteini. El piso de la cápsula está abierto, dejando ver el cuerpo glandular en su interior.

en su tercio anterior. La pared de la glándula es relativamente gruesa y no permite visualizar el interior. Dorsalmente, el tercio medio de la glándula se encuentra cubierto por una delgada membrana transparente, asociada y limitada posteriormente a las dos primeras ramas nerviosas provenientes de los ganglios cerebroides, mientras que el tercio posterior se encuentra libre, por su cara dorsal, en la cavidad visceral, pero sujeta al pie por escasas fibras desde sus bordes laterales.

El cuerpo glandular propiamente dicho está sujeto por proyecciones emitidas desde el techo y los lados del piso de la cápsula, que se presentan en número de 9 a 11. La superficie del cuerpo glandular es lisa, de color amarillento cremoso, y en el centro existe un surco longitudinal ancho y poco profundo que se extiende en el tercio anterior de la glándula (Fig. 4B). En sus bordes laterales se observan entre 9 y 11 aberturas grandes. En el interior existen acinos glandulares, los cuales están separados por las proyecciones del techo de la cápsula. El lumen ocupa todo el largo de la glándula y su ancho es reducido, pero es proporcionalmente mayor al lumen de $M$. lichtensteini y $M$. thammianus.

El piso de la cápsula es liso y presenta un levantamiento longitudinal que se extiende desde la abertura hasta la mitad del tercio anterior de la glándula (Fig. 4A); coincide con el surco de la parte glandular.

\section{Megalobulimus popelairianus (Figs. 5A y B)}

La glándula pediosa de $M$. popelairianus tiene forma de daga, alargada, deprimida y bordes irregulares. Su longitud $(24,19$ $\mathrm{mm}$ ) (Tabla 2) abarca casi toda la longitud de la cavidad visceral. $\mathrm{El}$ ancho mayor se ubica en el tercio anterior.

Dorsalmente, el tercio medio de la glándula se encuentra cubierto por una delgada y transparente membrana, asociada posteriormente a las dos primeras ramas nerviosas y limitada

\section{Cuerpo glandular}

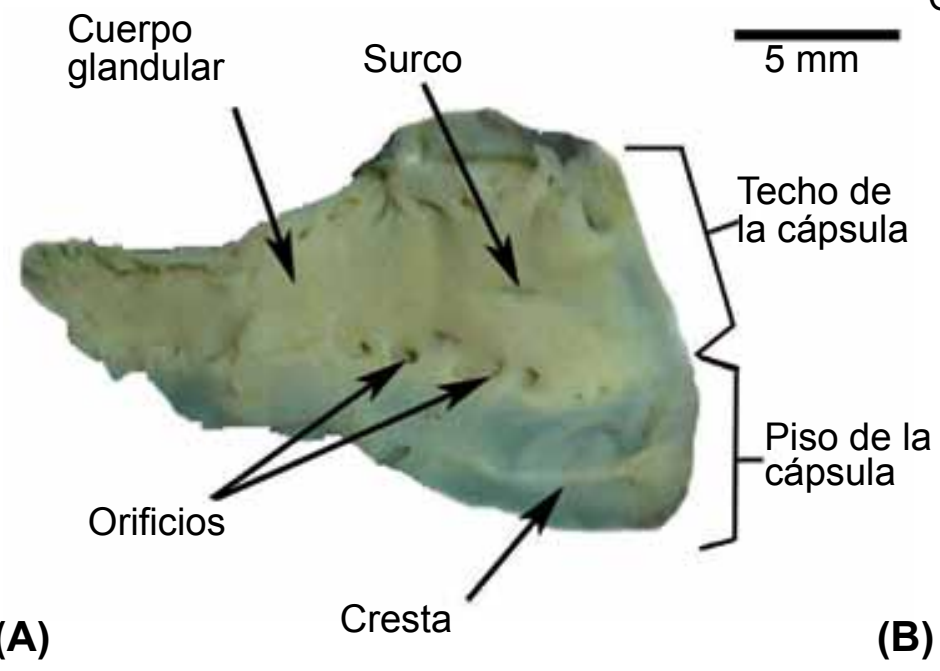

Figura 4. Glándula pediosa de Megalobulimus maximus. (A) Glándula abierta por la pared lateral, (B) Detalle de región anterior del cuerpo glandular. 


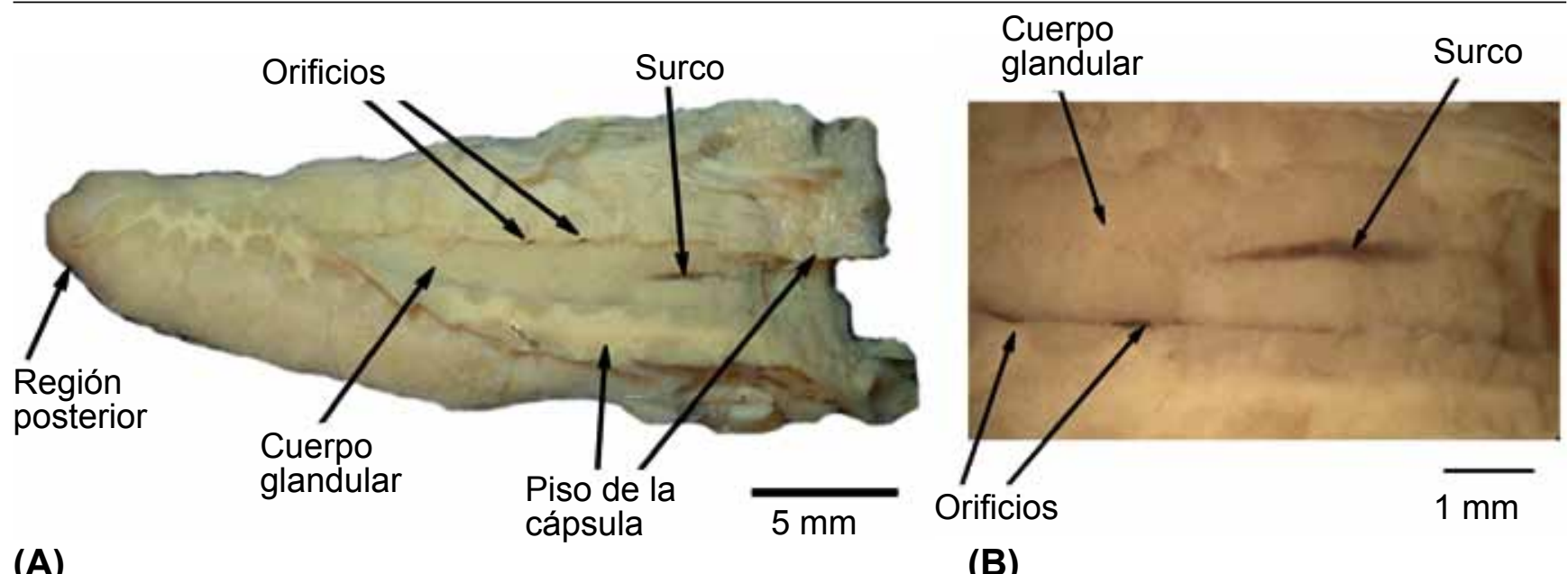

(A)

(B)

Figura 5. Glándula pediosa de Megalobulimus popelairianus en vista ventral. (A) Detalle del interior de la glándula con el piso de la cápsula abierto. (B) Detalle de la región anterior del cuerpo glandular

anteriormente por los dos haces musculares oblicuos ubicados por encima de su extremo anterior. Tales haces musculares son los más gruesos dentro de las cinco especies de Megalobulimus estudiadas. Su tercio posterior está libre en la cavidad visceral, por su cara dorsal, pero sujeta al pie por escasas fibras que provienen de sus bordes laterales. La cápsula de la glándula es más gruesa que la de $M$. maximus, que no permite apreciar su interior (Fig. 5A). El lumen es reducido, abarca toda la longitud de la glándula y es el más estrecho entre las cinco especies de Megalobulimus aquí descritas.

El cuerpo glandular propiamente dicho está sujeto por proyecciones emitidas del techo y de los lados del piso de la cápsula, las proyecciones del piso son proporcionalmente más cortas que en las otras especies de Megalobulimus, de modo que las aberturas (entre 10 y 13) a lo largo de sus bordes laterales colindantes con la cápsula se ven muy estrechas, a manera de hojales (Fig. 5B). La superficie del cuerpo glandular es lisa, de color amarillento cremoso, y en el centro existe un surco longitudinal que se extiende en el tercio anterior de la glándula. El interior del cuerpo glandular está formado por acinos glandulares separados por las proyecciones del techo de la cápsula.
El piso de la cápsula es liso y presenta un levantamiento de longitud variable $(5-14 \mathrm{~mm})$ a lo largo de la línea media de su porción anterior. Ésta coincide con el surco del cuerpo glandular.

\section{Megalobulimus thammianus (Fig. 6)}

La glándula pediosa de $M$. thammianus es alargada, deprimida y con bordes lisos. Su longitud (18,8 mm) (Tabla 2) abarca casi toda la cavidad visceral. El ancho es constante en casi todo su largo y presenta el extremo posterior romo. El tercio medio de la glándula se encuentra cubierto por un delgado tejido conectivo asociado a las ramas nerviosas, mientras que el tercio posterior, por su cara dorsal, se encuentra libre en la cavidad visceral. Escasas fibras la sujetan al pie, desde sus bordes laterales. La pared de la glándula es relativamente gruesa y no permite visualizar su interior.

El cuerpo glandular propiamente dicho es prominente con sólo parte de su cara ventral libre y el resto sujeto a la cápsula por proyecciones, por ende, el lumen es muy reducido. Las proyecciones ventrales son más gruesas y largas en la región anterior que en la posterior. Los puntos de inserción de las

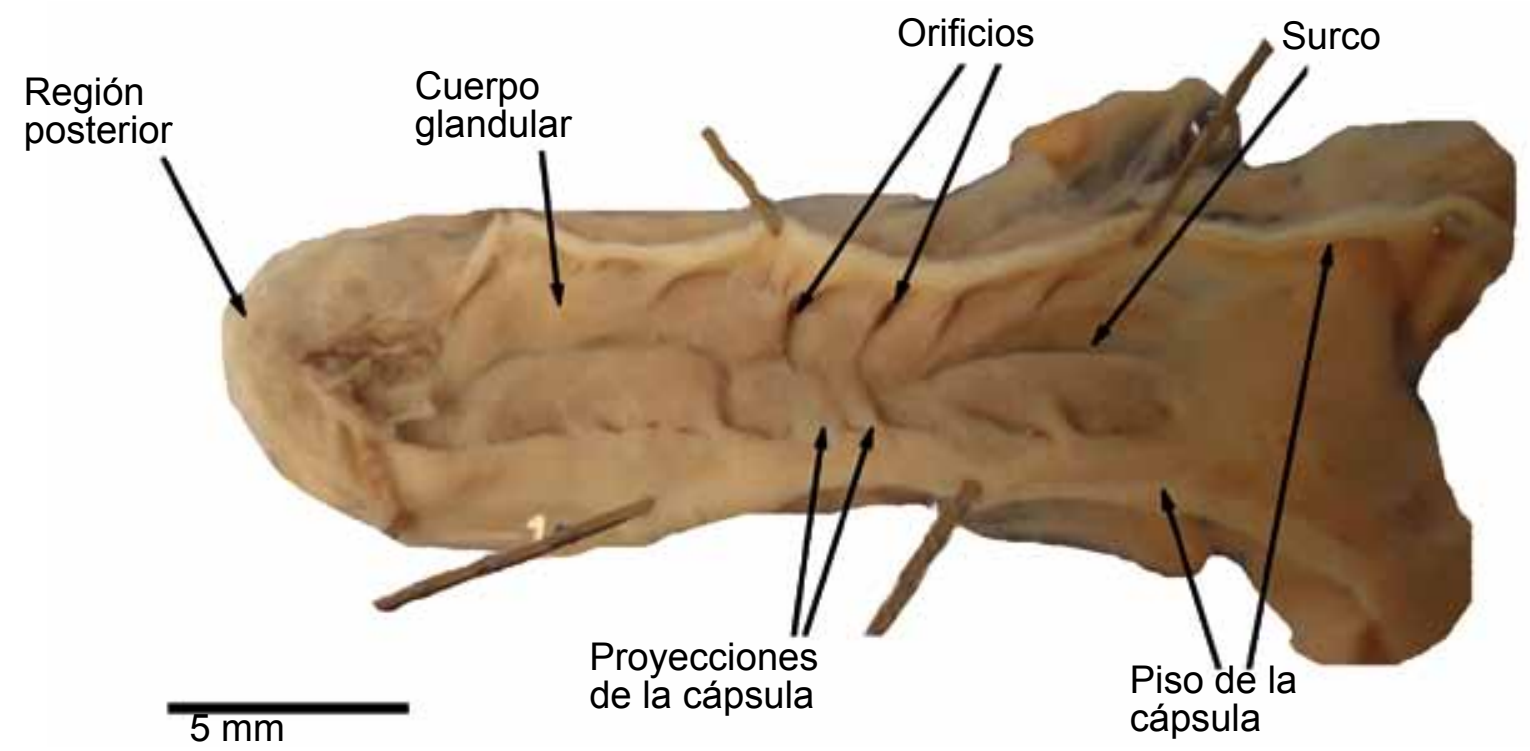

Figura 6. Glándula pediosa de Megalobulimus popelairianus var. thammianus Martens en vista ventral. 
proyecciones del piso se intercalan con los puntos de inserción de las proyecciones del techo, formando entre 8 y 13 aberturas estrechas, a lo largo de los bordes laterales colindantes con la cápsula. La superficie del cuerpo glandular es lisa, de color amarillento, en el centro existe un surco longitudinal que se extiende en el tercio anterior de la glándula. La profundidad del surco disminuye conforme avanza al extremo posterior. En uno de los especímenes el surco se presentó hasta el extremo posterior, interrumpido en la parte central (Fig. 6). En el interior se observan acinos glandulares. El piso de la cápsula es liso y no presenta el levantamiento longitudinal de las otras especies de Megalobulimus, sino un casi imperceptible engrosamiento en la línea media de su tercio anterior.

\section{Succinea peruviana (Fig. 7)}

La glándula pediosa de $S$. peruviana es alargada, deprimida cuya longitud (3,66 mm) (Tabla 2) abarca casi todo el piso de la cavidad visceral del animal. El ancho se mantiene constante en toda su longitud. El extremo de la región posterior es romo. Presenta una consistencia algodonada. Se encuentra sujeta por algunas fibras procedentes de sus bordes laterales. Dos haces musculares oblicuos pequeños y poco conspicuos, cubren parte del tercio anterior de la glándula.

La cápsula es bastante delgada exponiendo claramente los acinos glandulares, que le dan a la glándula su apariencia algodonosa. La parte glandular está adosada a la cápsula; en la mitad anterior se presenta como una capa delgada, mientras que en la mitad posterior la masa del cuerpo glandular se incrementa conformando un cuerpo más compacto. El lumen presenta un ancho constante hasta la mitad de la glándula en que disminuye drásticamente y se prolonga así hasta el extremo posterior. En el piso de la glándula se observa un surco conspicuo, angosto y profundo (Fig. 7). El techo es liso.

\section{Bostryx conspersus (Figs. 8A y B)}

La glándula pediosa en $B$. conspersus es alargada $(7,71 \mathrm{~mm})$ (Tabla 2), delgada, presenta bordes ligeramente lobulados y se extiende en casi toda la longitud de la cavidad visceral. La región anterior es ligeramente más ancha que la posterior. Su extremo posterior es romo. Su consistencia es algodonosa, como la de $S$. peruviana. Fibras la sujetan por sus lados al pie (Fig. 8a). Los dos
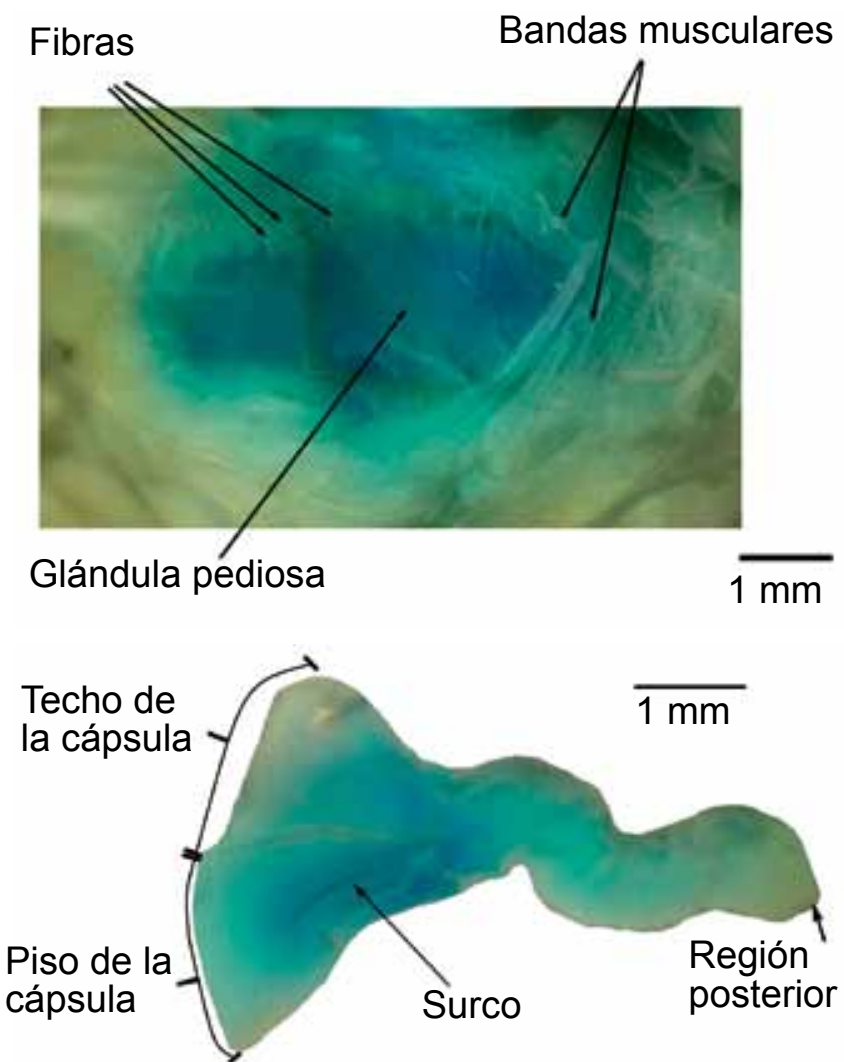

Figura 8. Glándula pediosa de Bostryx conspersus. (A) Detalles de la glándula sujeta al piso de la cavidad visceral, (B) Interior de la glándula abierta parcialmente a lo largo del lado izquierdo.

haces musculares transversales son conspicuos y cubren el tercio anterior de la glándula (Fig. 8A). Estos pueden ser levantados con facilidad por su parte media, mientras que los laterales se encuentran adheridos a la superficie dorsal de la glándula.

La cápsula es bastante delgada y transparente. El cuerpo glandular propiamente dicho está adosado a la cápsula; puede ser observado desde el exterior por la transparencia de la cápsula, y es la que le da a la glándula su apariencia algodononosa. En la mitad anterior de la glándula, el cuerpo glandular se presenta como una capa delgada, mientras que en la mitad posterior la masa del cuerpo glandular se incrementa conformando un cuerpo más compacto. El lumen es amplio en la mitad anterior de la glándula, en la mitad posterior el ancho se reduce bruscamente y continúa así hasta el extremo posterior. Se observa un surco

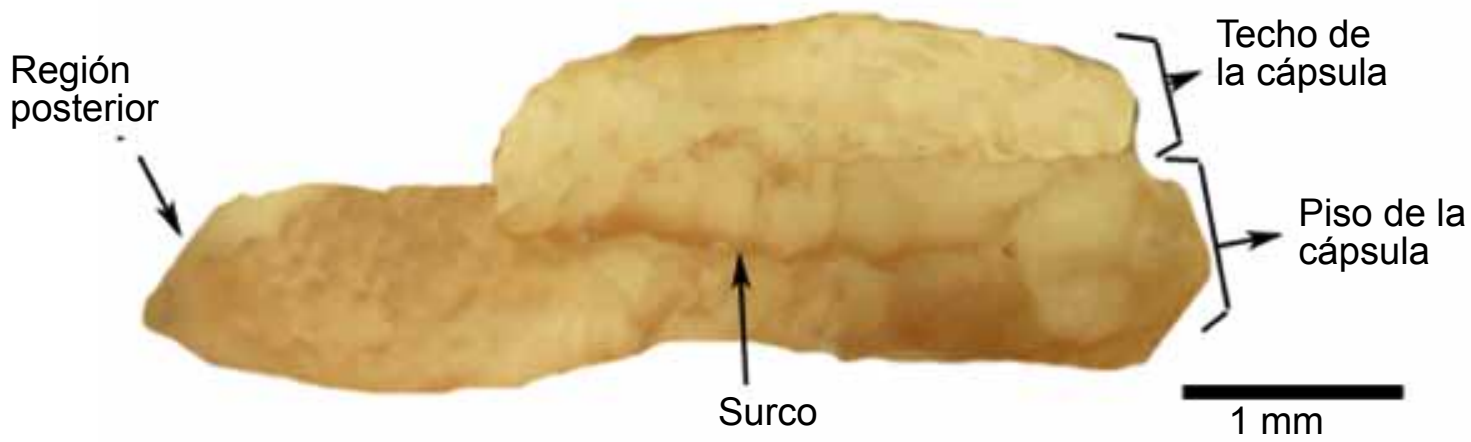

Figura 7. Interior de la glándula pediosa de Succinea peruviana abierta parcialmente por su lado derecho. 
conspicuo y poco profundo en la mitad anterior del piso de la glándula (Fig. 8B). El techo es liso.

\section{Cantareus aspersus (Figs. 9A y B)}

La glándula pediosa de $C$. aspersus está situada en la base de la cavidad visceral y no es vista inmediatamente luego del primer plano de disección, ya que está completamente aislada de la cavidad visceral, como si estuviera embebida en el pie (Fig. 9A). Una membrana muy delgada, conformada por numerosas fibras, se adosa a toda su superficie dorsal así como al pie. Los
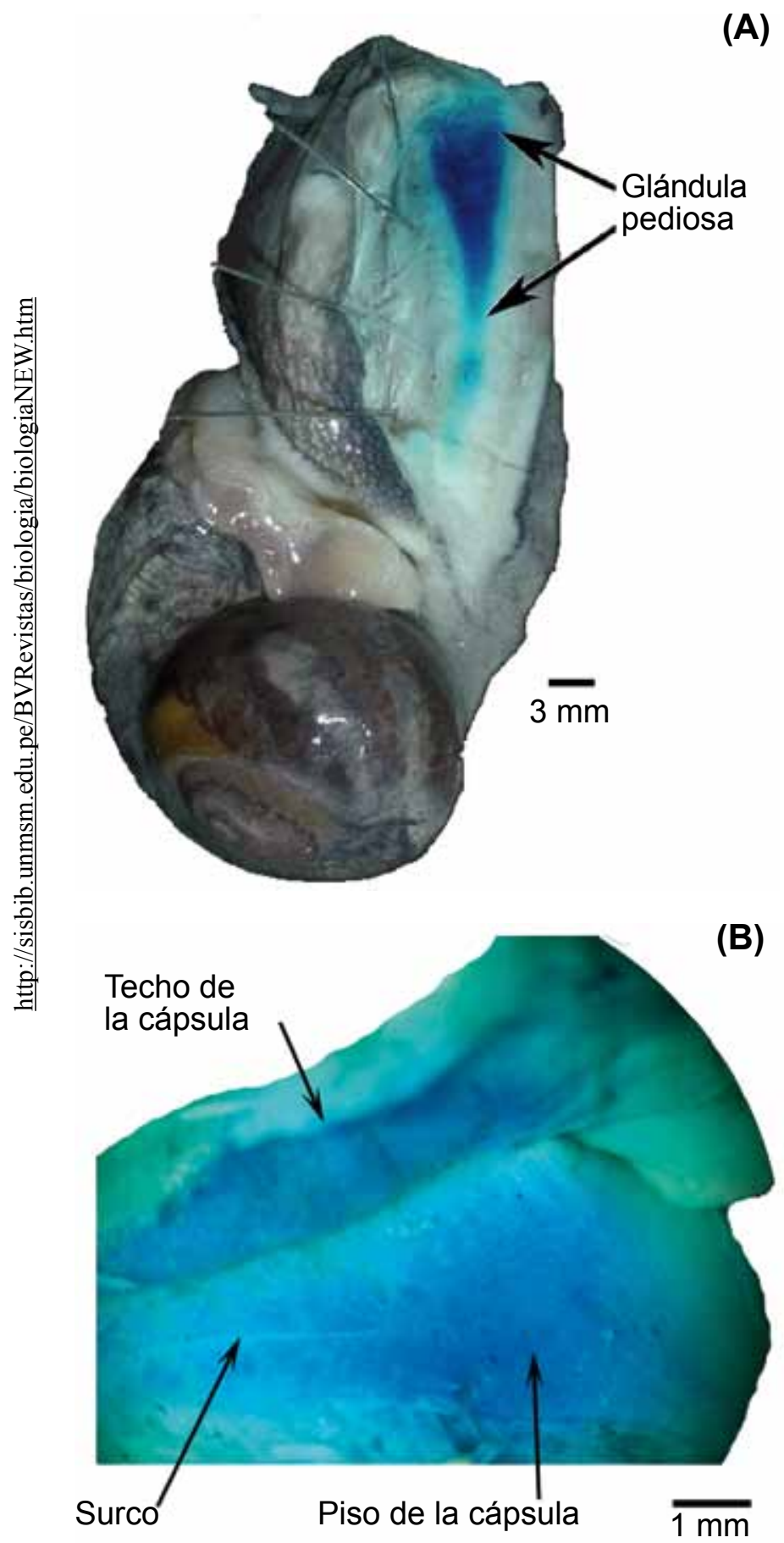

Figura 9. Glándula pediosa de Cantareus aspersus. (A) Vista del piso de la cavidad visceral. La glándula pediosa, inyectada con azul de metileno, se ubica debajo de una membrana que la separa de la cavidad. (B) Vista interna de la glándula pediosa, abierta a lo largo del lado derecho. dos haces musculares transversales oblicuos observados por encima de la región anterior de la glándula en otras especies, aqui son inconspícuos y están adheridos a la superficie dorsal de la glándula.

La glándula pediosa de $C$. cantareus no es compacta, para evidenciarla mejor fue necesario inyectarle azul de metileno. Es una estructura sacular, aplanada, se extiende en toda la longitud de la cavidad visceral. Su longitud promedio es $18,56 \mathrm{~mm}$ (Tabla 2). La cápsula es muy delgada y transparente, permite observar los acinos. El cuerpo glandular propiamente dicho está adosado a la cápsula; se presenta como una lámina delgada. El lumen es amplio y se extiende hasta casi el extremo posterior de la glándula. En la línea media de la mitad posterior del piso presenta un surco longitudinal que se prolonga hasta el extremo posterior de la glándula (Fig. 9B). Tanto el techo como el piso de la glándula presentan una superficie ligeramente rugosa.

\section{Heterovaginina limayana (Figs. 10A, B y C)}

La glándula pediosa de $H$. limayana es de apariencia vermiforme y su longitud promedio $(8,17 \mathrm{~mm})$ (Tabla 2$)$ corresponde a menos de la quinta parte del largo de la cavidad visceral. Existen unas delgadas fibras que sujetan la superficie ventral media de la glándula al pie, pero son tan pocas y delgadas que suelen pasar desapercibidas; fuera de esto, no existe otro tipo de unión con el pie. La región posterior de la glándula, junto con los ganglios cerebroides y el esófago, se encuentran cubiertos por abundante conectivo laxo semitransparente (Fig. 10A). Se presentan dos haces musculares oblicuos que van de lado a lado de la pared del cuerpo y cubren el tercio anterior de la glándula.

En la región anterior de la glándula se nota por transparencia una pequeña área triangular, con un vértice posterior, y sin apariencia secretora (Figs. 10B y C). La parte glandular propiamente dicha está adherida a toda la superficie interna de la cápsula.

\section{Discusión}

Dayrat y Tillier (2002) emplearon la glándula pedal como carácter para la reconstrucción filogenética de gastrópodos eutineuros; la presencia de una membrana que separa a la glándula pediosa de la cavidad visceral fue el carácter designado a todos los Stylommatophora, entre ellos Succinea y Cantareus. En el presente trabajo pudimos corroborar la existencia de una membrana propiamente dicha sólo en Cantareus aspersus. En el caso de las especies de Megalobulimus, Succinea y Bostryx aquí estudiadas, se observó un grado creciente de sujeción de la glándula pediosa al pie, sin llegar a formar en ningún caso una membrana de separación total. Barr (1926) enfatizó que en Milax sowerbii, otro estilomatóforo, la glándula pediosa no está embebida al pie sino que está sujeta a él por pequeños músculos. La sinapomorfía en los Systellommatophora es que la glándula está libre (Dayrat y Tillier 2002); en el presente trabajo se observó que efectivamente la glándula pediosa de Heterovaginina limayana no está separada de la cavidad visceral, ni sujeta al pie, pero tampoco es un órgano que flota libremente.

La porción glandular de la glándula pediosa en pulmonados terrestres es de estructura variable, como lo demostró André (1894) para diversas especies europeas. Para las cinco especies de Megalobulimus aquí estudiadas, la parte glandular es voluminosa y presenta un surco longitudinal medio, carácter observado sólo 
en otra especie de Megalobulimus (M. loretzianus) (Hylton-Scott 1939), por lo que podría constituir una sinapomorfía para el género Megalobulimus. Otro carácter observado en Megalobulimus es la presencia de un pequeńo levantamiento a lo largo de la línea media del piso de la cápsula, denominado "cresta" por (Hylton-Scott 1939). Por el contrario, en Bostryx conspersus, Succinea peruviana y Cantareus aspersus, colectados en el Departamento de Lima (Perú), se observa un surco de longitud variable en la línea media del piso de la cápsula, carácter que fue descrito para Cantareus aspersus de Europa por André (1894).

En la filogenia molecular de los Stylommatophora (Wade et al. 2006), los Orthalicoidea (Bostryx) y Elasmongnata (Succinea) forman un clado; esa relación evolutiva cercana parece estar reflejada también en la glándula pediosa. Se ha encontrado similitud en la distribución del cuerpo glandular, el lumen y el grado de sujeción de la glándula pediosa al pie de Bostryx conspersus y Succinea peruviana.

\section{Región}

posterior
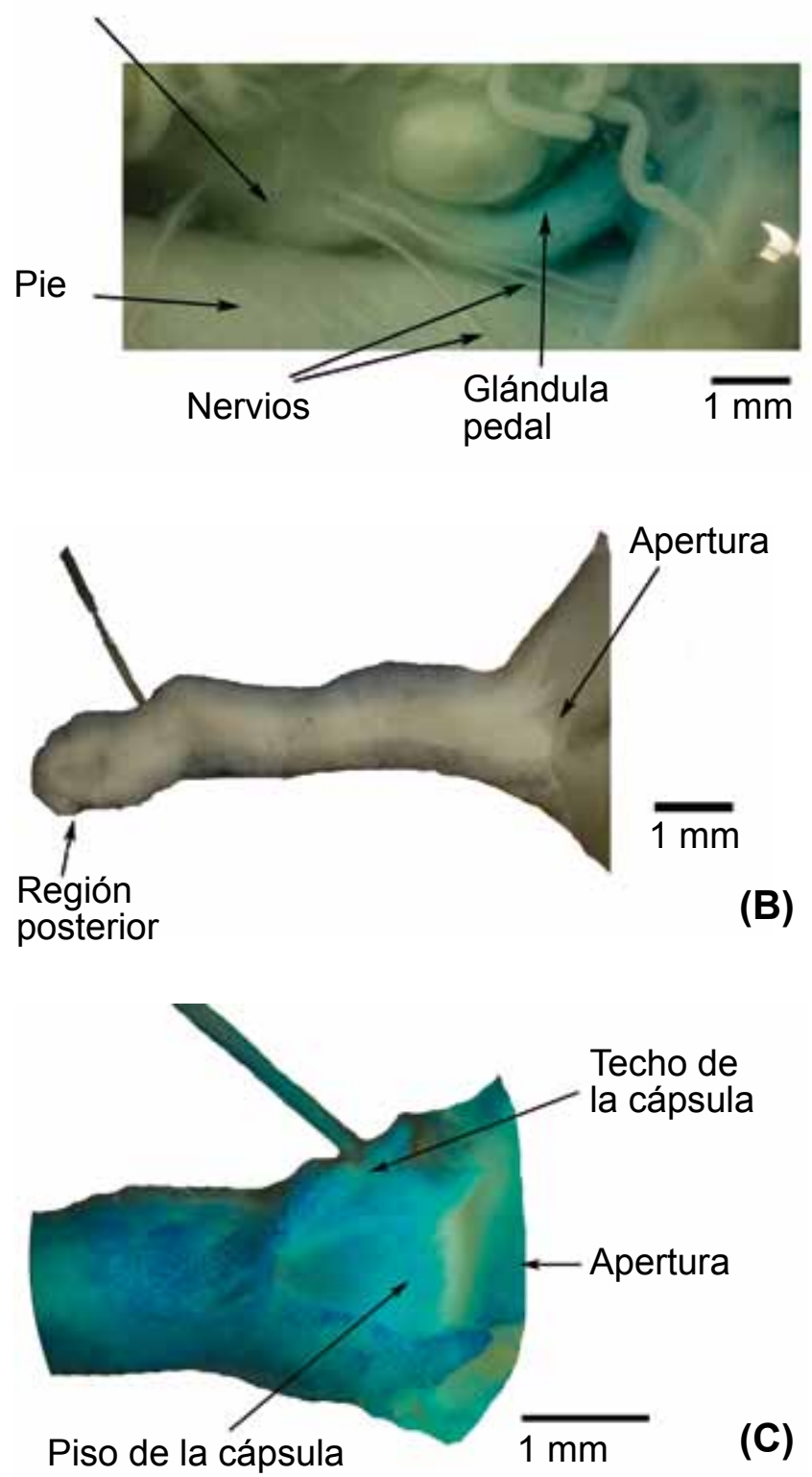

Figura 10. Glándula pediosa de Heterovaginina limayana. (A) Posición de la glándula en la cavidad visceral, (B) Vista dorsal de la glándula, (C) Vista interna de la porción anterior de la glándula.
Las diferencias macroscópicas de las glándulas pediosas de las especies aquí estudiadas se correlacionarían con diferencias a nivel histológico y con las características químicas de la baba, cuyas diferencias a nivel interespecífico ya han sido observadas en otros caracoles pulmonados (Skingsley 2000).

\section{Agradecimientos}

El material biológico utilizado en el presente trabajo fue obtenido en la realización de proyectos financiados por la Universidad Nacional Mayor de San Marcos (VRI-PEM2007B28; VRI-CSI-091001041) y la ONG Inka Terra Asociación. Agradecemos al Dr. W.E. Duellman, Director del Progama BIOTROP, Universidad de Kansas, Laurence, USA, por el apoyo brindado en Cuzco Amazónico. Al INRENA por los permisos para trabajo en áreas reservadas. A J. Purisaca y $\mathrm{H}$. Méndez por el apoyo logístico en INKATERRA, a C. Calderón y L. Ruiz en San Martín y a F. Ramírez, N. Medina y T. Ramos en las lomas de Lima. Quedamos agradecidos a C. Díaz por el material biológico cedido; a M. Quispe y D. Fernández por el cuidado de los caracoles vivos y a J. Ramírez por facilitarnos material bibliográfico.

\section{Literatura citada}

André E. 1894. Recherches sur la glande pedieuse des Pulmones. Rev. Suisse Zool. 2 : 291-353.

Barker G.M. 2001. Gastropods on Land: Phylogeny, Diversity and Adaptive Morphology. In G.M. Barker, eds. The biology of terrestrial molluscs. CABI Publishing, Oxon, UK. Pp.1-146.

Barr R.A. 1926. Some Observations on the Pedal Gland of Milax. Quarterly Journal of Microscopical Science 70(2): 647667.

Beaumont A. \& P. Cassier, 1970. Travaux pratiques de Biologie animale. DUNOD. 480pp.

Cáceres P.A. 2006. Estudio comparativo de la Capacidad Humectante de la piel de activos cosméticos naturales respecto al aceite de Emú utilizando el Corneometer ${ }^{\circledR}$ CM 825. Memoria para obtener el Título de Químico Farmacéutico de la Universidad de Chile. Santiago, Chile. 61pp.

Chandra K. 1962. Morphogenesis of the pedal gland in the giant land snail Achatina fulica Bowdich. Current Science 32: 167-168.

Dayrat B. \& S. Tillier. 2002. Evolutionary relationships of euthyneyran gastropods (Mollusca): a cladistic re-evaluation of morphological characters. Zoological Journal of the Linnean Society 135: 403-470.

Dayrat B. \& S. Tillier. 2003. Goals and limits of phylogenetics: the euthyneuran gastropods. In C. Lydeard and D. R. Lindberg, eds. Molecular Systematics and Phylogeography of Mollusks. Smithsonian Institution, Washington, DC. Pp. 161-184.

Hylton-Scott, M.L. 1939. Estudio anatómico de Borus "Strophocheilus lorentzianus" (Doer) (Mol. Pulm.). Revista del Museo de La Plata, nueva serie, 1(7): 217-278.

Levene P.A. 1925. The mucoproteins of the snails, Helix aspersa, Helix pomatia. J. biol. Chem. 65: 683-700.

Pena M.S., N.C. Salgado \& A.C.S. Coelho. 2004. Recharacterization of Strophocheilus miersi Da Costa (Mollusca, Pulmonata, Strophocheilidae). Rev. Bras. Zool. 21(1): 45-50.

Ramírez R. \& S. Cáceres. 1991. Caracoles terrestres (Mollusca, Gastropoda) comestibles en el Perú. Boletín de Lima (77): 67-74. 
BORDA ET AL.

Skingsley D.R., A.J. White \& A. Weston. 2000. Analysis of pulmonate mucus by Infrared Spectroscopy. J. Mollus. Stud. 66: 363-372.

Thomé J.W. \& V.L.R. Lopes. 1973. Aulas práticas de Zoologia, I. Dissecação de um molusco gastrópode desprovido de concha. Iheringia 3: 34-45.
Thomé J.W., A.N. Quillfeldt, M.T. Osorio, P.T. Campos \& V.I. Schneider. 1994. Manual de aulas prácticas de zoologia: estudo morfo-anatômico de um Molusco Gastrópode Mesuretra. EDIPUCRS - Serie Zoologia. 32pp.

Wade C., P. Mordan \& F. Naggs. 2006. Evolutionary relationships among the Pulmonate land snails and slugs (Pulmonata, Stylommatophora). Biological Journal of the Linnean Society 87(4): 593-610. 